\title{
Exploring the mechanism of Bushenhuoxue formula acting on postmenopausal osteoporosis via network pharmacology and experimental validation
}

\section{Chunhai Ke}

Ningo Medical Center Lihuili Hospital

\section{Yizhou Chen}

Ningbo Yinzhou No. 2 Hospital

\section{Yi Wang}

Ningbo Hospital of Traditional Chinese Medicine

\section{Guonong He}

Ningbo Hospital of Traditional Chinese Medicine

\section{Hongkai Lou}

Ningbo Hospital of Traditional Chinese Medicine

\section{Dandan Mao}

Ningbo Hospital of Traditional Chinese Medicine

\section{Hongting Jin}

the First Affiliated Hospital of Zhejiang Chinese Medical University

\section{Chengliang Wu}

the First Affiliated Hospital of Zhejiang Chinese Medical University

\section{Liang Fang}

the First Affiliated Hospital of Zhejiang Chinese Medical University

Chenjie Xia ( $\square$ xiachenjie@zcmu.edu.cn )

Ningo Medical Center Lihuili Hospital

\section{Research Article}

Keywords: Bushenhuoxue formula, Network pharmacology, Postmenopausal osteoporosis, Angiogenesis, Osteogenesis, $\beta$-catenin

Posted Date: February 17th, 2021

DOl: https://doi.org/10.21203/rs.3.rs-156672/v1

License: (c) (1) This work is licensed under a Creative Commons Attribution 4.0 International License. Read Full License 


\section{Abstract}

Background: Bushenhuoxue (BSHX) formula, a ten-compound herbal decoction, has been used to treat postmenopausal osteoporosis (PMOP) in China for decades. Our previous study also revealed the antiPMOP effects of BSHX formula in ovariectomized (OVX) mice. However, its pharmacological mechanisms remain unknown.

Methods: Network pharmacology followed by animal experiments was used to explore the action mechanism of BSHX formula. Firstly, we obtained the chemical compounds and potential targets of BSHX formula using TCMSP and TCMID databases. Simultaneously, GeneCards and DisGeNET databases were used to determine the targets in PMOP. Based on their overlapping genes between BSHX formula and PMOP, a protein-protein interaction (PPI) network was constructed for screening hub-targets. GO and KEGG enrichment analyses were further performed to identify the critical biological processes and signaling pathways. In vivo experimental study, an OVX mouse model was established to determine the anti-PMOP effects of BSHX formula via Micro-CT assay and ABH staining. The expressions of $\beta$ catenin, alkaline phosphatase (ALP), vascular endothelial growth factor (VEGF) and CD31 were detected by immunohistochemistry.

Results: A total of 218 active ingredients and 274 related targets were identified in BSHX formula. After matching with 292 therapeutic targets of PMOP, 64 overlapping genes were obtained and further used to build a PPI network in which CTNNB1 and VEGFA played a central role. GO and KEGG enrichment analyses indicated that the anti-PMOP effects of BSHX formula were mainly associated with cell proliferation, angiogenesis and their regulated pathways including $\beta$-catenin signaling and VEGF signaling. In mouse experiments, we revealed that bone mass and blood vessels in the OVX mice were significantly enhanced after treated with BSHX formula for 8 weeks. Moreover, down-regulations of $\beta$ catenin, ALP, VEGF and CD31 caused by OVX surgery were significantly restored by BSHX formula.

Conclusions: Through network pharmacology and an experimental validation, we have revealed that BSHX formula exerts anti-PMOP effects mainly through promoting $\beta$-catenin-mediated osteogenesis and VEGF-mediated angiogenesis. BSHX formula can be considered as a new option for the treatment of PMOP.

\section{Introduction}

Postmenopausal osteoporosis (PMOP) is a common bone disorder that was characterized by low bone mineral density and microstructure deterioration ${ }^{1}$. It is estimated that more than $15 \%$ of postmenopausal women over 50 years old are suffering from PMOP in United States ${ }^{2}$, which can cause poor clinical outcomes including low back pain, hunchback and fragility fractures ${ }^{3}$. Although there exit many antiosteoporosis drugs such as active vitamin D, estrogen receptor modulators, bisphosphonates and parathyroid hormone ${ }^{4}$, their application and efficacy remain limited because of various undesirable 
effects $^{5}$. Currently, natural productions and herbs attract more and more attentions for their potential antiosteoporosis effects and relative safety ${ }^{6}$.

Traditional Chinese medicine (TCM) is used as a main alternative treatment worldwide. Bu-shen-huo-xue (BSHX) formula is a Chinese herbal decoction composed by ten herbs ${ }^{7}$. In China, BSHX formula has been used to treat PMOP since a long time, and gains satisfactory outcomes. Our recent study found that BSHX formula can attenuate bone loss in ovariectomized (OVX) mice, confirming its anti-PMOP effects ${ }^{8}$. The theory of "kidney governing bones" has well clarified its positive effects on PMOP in TCM perspective. Bone loss in PMOP is due to deficiency of kidney-qi and BSHX formula strengthens bone through tonifying kidney-qi ${ }^{9}$. However, it is still unclear about the pharmacological mechanism of BSHX formula.

For the multi-component properties of TCM formula, conventional animal or cellular research strategies can not meet the requirements to study massive molecular targets simultaneously. Network pharmacology is an emerging discipline that integrates pharmacology, bioinformatics, system biology and computer science ${ }^{10,11}$. Based on the concept of "Medicine - Target - Gene - Disease", network pharmacology provides a systematic and integrative viewpoint to explore the relationship between TCM formula and diseases ${ }^{12,13}$. Here, through network pharmacology, we comprehensively predicted the underlying molecular targets of BSHX formula in the treatment of PMOP.

In the network pharmacology study, related ingredients of BSHX formula originated from two databases were used to fish the potential targets. After matching with PMOP therapeutic targets obtained from GeneCards and DisGeNET databases, 64 overlapping target genes were identified and further used to build a protein-protein (PPI) network for analyzing their internal interactions. DAVID database and Kyoto Encyclopedia of Genes and Genomes (KEGG) database were utilized for gene ontology (GO) and pathway enrichment analysis, respectively. Based on the results of network pharmacology, animal experiments were performed to validate the key biological process and signaling pathway through which BSHX formula exerts the anti-PMOP effects.

\section{Methods And Materials}

\subsection{Components and preparation of BSHX formula}

BSHX formula is composed of 10 herbs (Table 1) that were provided by the first affiliate hospital of Zhejiang Chinese Medical University (Hangzhou, China). The extraction process of these herbs were performed as previously described ${ }^{14}$. Eucommia ulmoides (Oliv.), Cornus officinalis (Sieb.), Glycyrrhiza uralensis (Fisch.), Aconitum carmichaeli (Debx.), Lycium barbarum (L.), Carthamus tinctorius (L.), Rehmannia glutinous (Liboscb) and Dioscoreae opposite (Thunb.) were mixed and extracted with water at a ratio of 2:1:1:2:1:1:3:2. Other two herbs, Cinnamomum cassia (Presl) and Prunus persica (Batsch.) were mixed at a ratio of 2:1 for ethanol extraction. These two parts were mixed and concentrated to $2 \mathrm{~g}$ crude drug/mL for intragastric administration. 
Table 1

The compositions of BSHXF.

\begin{tabular}{|c|c|c|c|c|}
\hline $\begin{array}{l}\text { Chinese name } \\
\text { (abbreviation) }\end{array}$ & Botanical name & Common name & Parts used & Proportion \\
\hline $\begin{array}{l}\text { Shu Di Huang } \\
\text { (SDH) }\end{array}$ & Rehmannia glutinosa(Liboscb) & Rehmannia & Root & $17.40 \%$ \\
\hline $\begin{array}{l}\text { Du Zhong } \\
\text { (DZ) }\end{array}$ & Eucommia ulmoides(Oliv.) & Eucommia & Bark & $11.80 \%$ \\
\hline $\begin{array}{l}\text { Fu Zi } \\
\text { (FZ) }\end{array}$ & Aconitum carmichaeli(Debx.) & Monkshood & Root & $11.80 \%$ \\
\hline $\begin{array}{l}\text { Gou Qi Zi } \\
\text { (GQZ) }\end{array}$ & Lycium barbarum(L.) & Matrimony Vine & Fruit & $11.80 \%$ \\
\hline $\begin{array}{l}\text { Rou Gui } \\
\text { (RG) }\end{array}$ & Cinnamomum cassia(Presl) & Cassia Bark Tree & Bark & $5.90 \%$ \\
\hline $\begin{array}{l}\text { Shan Zhu Yu } \\
\text { (SZY) }\end{array}$ & Cornus officinalis(Sieb.) & Medical Dogwood & Fruit & $5.90 \%$ \\
\hline $\begin{array}{l}\text { Tao Ren } \\
\text { (TR) }\end{array}$ & Prunus persica(Batsch.) & Peach & Fruit & $11.80 \%$ \\
\hline $\begin{array}{l}\text { Hong Hua } \\
(\mathrm{HH})\end{array}$ & Carthamus tinctorius(L.) & False Saffron & Corolla & $5.90 \%$ \\
\hline $\begin{array}{l}\text { Shan Yao } \\
\text { (SY) }\end{array}$ & Dioscoreae opposite(Thunb.) & Common Yam & Root & $11.80 \%$ \\
\hline $\begin{array}{l}\text { Gan Cao } \\
\text { (GC) }\end{array}$ & Glycyrrhiza uralensis(Fisch.) & Ural Licorice & Root & $5.90 \%$ \\
\hline
\end{tabular}

\subsection{Identification and target prediction of active ingredients}

Traditional Chinese Medicine System Pharmacology Database (TCMSP ${ }^{\mathrm{TM}}$, http://lsp.nwu.edu.cn/tcmsp.php) and Traditional Chinese Medicines Integrated Database (TCMID, http://119.3.41.228:8000/) were used to search the chemical ingredients of BSHX formula. Oral bioavailability $(O B \geq 30 \%)$ and drug-like $(D L \geq 0.18)$ were set as the screening threshold ${ }^{15}$, and a total of 
218 chemical ingredients were identified in this formula. UniProt database (https://www.uniprot.org/) was used to predict the target genes for these active ingredients.

\subsection{PMOP targets screening}

GeneCards Database (https://www.genecards.org/) and DisGeNET Database (https://www.disgenet.org/) were used to collect PMOP-associated target genes. After screening with the filter of score $>10$ for GeneCards Database and score $>0.1$ for DisGeNET Database (Zhu and Hou 2020), a total of 292 therapeutic targets were identified. Then, a Venn diagram was constructed to determine the overlapping genes between PMOP and active ingredients, which were terms as the potential therapeutic targets of BSHX formula for PMOP treatment.

\subsection{Network construction}

\subsubsection{Herb-Ingredient and PPI networks construction}

The network was constructed as follows: (1) The active ingredients obtained from BSHXF via TCMSP database and TCMID database were imported into Cytoscape3.8.0 software (http://www.cytoscape.org/) to establish a Herb-Ingredient network; (2) The overlapping targets were analyzed using the String database (https://string-db.org/) to build a PPI network. The PPI network topological feature was evaluate by three topological characteristics: Degree Centrality (DC), Betweenness Centrality (BC) and Closeness Centrality (CC).

\subsubsection{Gene ontology and pathway enrichment analyses}

The gene ontology (GO) enrichment analysis including biological process (BP), cellular component (CC), and molecular function (MF) terms were performed on the 64 overlapping targets using DAVID database (version 6.8, https://david.ncifcrf.gov/home.jsp). KEGG database (https://www.kegg.jp/) was used to identify the potential signaling pathways.

\subsection{Experimental groups and OVX model}

Ten-week-old female C57BL/6 J mice provided by Shanghai Laboratory Animal Center of Chinese Academy of Science (Shanghai, China) were randomly divided into three groups ( $n=8$ in each group): the sham group, the OVX group and the BSHX formula group. Mice in the latter two groups were subjected to bilateral ovariectomy, whereas a sham surgery only extracting the equal surrounding fatty tissues of ovaries were performed in the sham ones. BSHX formula was orally administered to the mice in BSHX formula group at a dosage of $0.2 \mathrm{~mL} / 10 \mathrm{~g}$ body weight every two days ${ }^{8}$. The mice in the sham group and the OVX group were given same dosage of $0.9 \%$ normal saline. All animal experiments were approved by the Animal Ethics Committee of Zhejiang Chinese Medical University (LZ12H27001).

\subsection{Micro-CT analysis}

After administration with BSHX formula for 8 weeks, femur samples were obtained from the mice in each group for micro-computed tomography (Micro-CT) analysis. Three-dimensional (3D) images of femoral metaphysis were reconstruct using NRecon software. The parameters of bone microstructure including 
bone volume fraction (BV/TV, \%), average trabecular number (Tb. N, 1/mm), average trabecular thickness (Tb. Th, $\mathrm{mm}$ ), average trabecular separation (Tb. Sp, $\mathrm{mm}$ ) and bone mineral density (BMD) was calculated as previously described ${ }^{16}$.

\subsection{Histology and immunohistochemistry (IHC)}

After Micro-CT scanning, femur samples were processed for paraffin sections at the thick of $3 \mu \mathrm{m}$ as previously described ${ }^{17}$. Alcian Blue Hematoxylin/Orange G (ABH) staining were performed on these sections for morphological analysis. The numbers of blood vessel and trabecular area (\%) in the region of interest were measured using OsteoMetrics software (Decatur, GA, USA) by two researchers. The IHC assay were detected as follows: 1 . sections were treated with $0.01 \mathrm{M}$ citrate buffer (Solarbio, Beijing, CN) at $60^{\circ} \mathrm{C}$ for $4 \mathrm{~h}$ as antigen retrieval; 2 . sections then were incubated in primary antibodies of CD31 (Diagbio, AGR52748, CN), VEGF (ARIGO, ARG10513, CN), ß-catenin (abcam, ab32572, UK), alkaline phosphatase (ALP, ARIGO, ARG57422, CN) overnight at $4^{\circ} \mathrm{C}$; 3 . sections were incubated in secondary antibodies for 20 min and diaminobenzidine (DAB) solution for 1 min to detect positive staining; 4 . sections were counterstained with hematoxylin. Image_J software was used to analyzed the quantification of positive staining.

\subsection{Statistical analysis}

All data were presented as mean \pm standard deviation. One-way ANOVA followed by Dunnett's test were performed using SSPS 20.0 software. $P<0.001$ was considered as statistical significance.

\section{Results}

\subsection{Active ingredients of BSHX formula}

After screening the TCMID and TCMSP databases with the ADME thresholds of $\mathrm{OB} \geqq 30 \%$ and $D L \geqq 0.18$, a total of 218 related ingredients were identified in BSHX formula, including 87 ingredients $(40.0 \%)$ in GC, $36(16.5 \%)$ in GQZ, 26 (11.9\%) in DZ, 18 (8.2\%) in TR, 17 (7.8\%) in HH, 14 (6.4\%) in SZY, 12 (5.5\%) in SY, 5 $(2.3 \%)$ in $\mathrm{FZ}, 2(0.9 \%)$ in SDH, $0(0 \%)$ in RG. As shown in Fig. 1, a Herb-Ingredient network were further constructed using Cytoscape software. According to the descending order of edge number in the HerbIngredient network, we listed and analyzed the top four ingredients that were beta-sitosterol (MOL000358, $\mathrm{DL}=0.75, \mathrm{OB}=36.91$, found in DZ, GQZ, HH, TR, SZY), stigmasterol (MOL000449, DL = 0.76, OB = 43.83, found in GQZ, SY, SDH, HH, SZY), quercetin (MOL000098, DL = 0.28, OB = 46.43, found in DZ, GC, GQZ, $\mathrm{HH}$ ) and sitosterol (MOL000359, DL = 0.75, OB = 36.91, found in $F Z, G C, S Z Y, S D H)$.

\subsection{Target prediction and PPI network analysis}

Target fishing on the 218 active ingredients was conducted in Uniprot databases, and we obtained 274 related targets of BSHX formula among which there were 220 in GC, 206 in DZ, 201 in HH, 190 in GQZ, 67 in SY, 46 in TR, 51 in SZY, 10 in, FZ 29 in SDH. Then, 292 PMOP related targets were obtained through 
GeneCards and DisGeNet databases. After the establishment of Venn diagram, 64 overlapping genes between BSHX formula and PMOP were identified and considered as the key therapeutic targets through which BSHX formula exerts the anti-PMOP effects (Fig. 2).

A Protein-Protein Interaction (PPI) network of these 64 overlapping genes was built in the the String database, which contained 64 nodes and 1110 edges (Fig. 3). Then, we used three main parameters, "degree", "betweenness" and "closeness", as the screening thresholds to select the central target genes. After the first screening round of degree $\geq 12$, betweenness $\geq 0.002$ and closeness $\geq 0.566,41$ nodes and 634 edges were obtained. Through the second screening round of degree $\geq 24$, betweenness $\geq 0.008$ and closeness $\geq 0.700$, only 21 nodes and 202 edges were identified (Fig. 4). The information of these hub nodes were listed in Table 2. 
Table 2

Information of 21 hub targets.

\begin{tabular}{|lll|}
\hline Uniprot ID & Gene Symbol & Description \\
\hline P31749 & AKT1 & RAC-alpha serine/threonine-protein kinase \\
\hline P15692 & VEGFA & Vascular endothelial growth factor A \\
\hline P01375 & TNF & Tumor necrosis factor \\
\hline P27361 & MAPK3 & Mitogen-activated protein kinase 3 \\
\hline P14780 & MMP9 & Matrix metalloproteinase-9 \\
\hline P04637 & TP53 & Cellular tumor antigen p53 \\
\hline P05412 & JUN & Transcription factor AP-1 \\
\hline P01133 & EGF & Pro-epidermal growth factor \\
\hline P35354 & PTGS2 & Prostaglandin G/H synthase 2 \\
\hline P40763 & STAT3 & Signal transducer and activator of transcription 3 3 \\
\hline P05121 & SERPINE1 & Plasminogen activator inhibitor 1 \\
\hline P22301 & IL10 & Interleukin-10 \\
\hline P01584 & IL1B & Interleukin-1 beta \\
\hline P10145 & CXCL8 & Interleukin-8 \\
\hline P35222 & CTNNB1 & Catenin beta-1 \\
\hline P37231 & PPARG & Peroxisome proliferator-activated receptor gamma \\
\hline P01100 & FOS & Proto-oncogene c-Fos \\
\hline Q15848 & ADIP0Q & Adiponectin \\
\hline P03372 & ESR1 & Estrogen receptor \\
\hline P02741 & CRP & C-reactive protein \\
\hline P28482 & MAPK1 & Mitogen-activated protein kinase 1 \\
\hline
\end{tabular}

\subsection{GO enrichment analysis}

Go enrichment analysis was performed on these 64 overlapping genes by using DAVID database. Based on the filter of FDR $<0.01$, a total of $55 \mathrm{GO}$ items were obtained, including $38 \mathrm{BP}$ terms, $4 \mathrm{CC}$ terms and 13 MF terms (Fig. 5A). As BP played a dominant role, we further build a bubble diagram for these BP terms 
according to the ascending order of log P-value (Fig. 5B). The results of diagram showed that $38 \mathrm{BP}$ were mainly concentrated in two categories, angiogenesis and cell proliferation. There are $6 \mathrm{BP}$ terms in the category of angiogenesis, including positive regulation of angiogenesis (G0:0045766), angiogenesis (G0:0001525), cellular response to hypoxia (GO:0071456), positive regulation of endothelial cell proliferation (G0:0001938), response to hypoxia (G0:0001666) and positive regulation of vascular endothelial growth factor production (G0:0010575). For cell proliferation, we found $4 \mathrm{BP}$ terms that were positive regulation of cell proliferation (G0:0008284), negative regulation of cell proliferation (GO:0008285), positive regulation of smooth muscle cell proliferation (G0:0048661) and positive regulation of endothelial cell proliferation (GO:0001938).

\subsection{KEGG enrichment analysis}

To determine the potential pathways of the anti-PMOP effects of BSHX formula, KEGG enrichment analysis was conducted on the 64 overlapping genes. Based on the threshold of Number $\geq 6$, we screened a total of 99 pathways (20 of these listed in Fig. 6), among which VEGF signaling (hsa04370) directly regulates angiogenesis and $\beta$-catenin signaling (hsa04310) controls cell proliferation.

\subsection{BSHX formula preserves bone mass in OVX mice}

To further carry out an animal experimental validation, C57BL/ $6 \mathrm{~J}$ mice were subjected to an OVX surgery and continuously treated with BSHX formula for 8 weeks. The 3D images of $\mu \mathrm{CT}$ showed severe bone loss in the OVX mice compared to the sham ones, and BSHX formula effectively alleviated bone loss (Fig. 7A). We also found that bone microstructure parameters were significantly improved after treatment of BSHX formula, including the increase of BMD, BV/TV, Tb.Th and Tb.N and the decrease of Tb.Sp (Fig. 7B-7F). These results indicated that BSHX formula could preserve bone mass in the OVX mice.

\subsection{BSHX formula promotes bone and blood vessel formation in OVX mice}

The pathological staining showed that the OVX mice presented sparse and thin trabeculae, massive lipid droplets and decreased blood vessels in the bone marrow (Fig. 8A, boxed areas a-c). After treated with BSHX formula for 8 weeks, both trabecular bone area and blood vessel number were significantly increased, while the area of lipid droplet was drastically decreased (Fig. 8B-8D). Bone homeostasis requires the balance of osteoblast-mediated bone formation and osteoclast-mediated bone resorption (Tella and Gallagher 2014). Our previous study have revealed that BSHX formula has little effect on osteoclasts, and the enhanced trabecular bone are relayed mainly on the osteoblastic activities (Xia et al. 2020). Blood vessels contain a layer flattened endothelial cells that not only participate in angiogenesis but also can regulate osteoblast proliferation for bone formation (Tong et al. 2019). Combined with the data of GO enrichment analysis, we found that BSHX formula prevented bone loss mainly through promoting osteoblast proliferation and angiogenesis.

\subsection{OVX-induced down-regulation of $\beta$-catenin, ALP, VEGF and CD31 are restored by BSHX formula} Furthermore, we evaluated the expressions of $\beta$-catenin signaling and VEGF signaling that have been identified by KEGG enrichment analysis. CD31, expressed in vascular endothelial cells, can specifically reflect the formation of blood vessel. ALP, synthesized by osteoblasts, plays a fundamental role in 
osteogenesis. The levels of $\beta$-catenin, ALP, VEGF and CD31, were significantly deceased in the OVX mice compared to the sham ones (Fig. 9A-9D). Mice treated with BSHX formula presented the improvement of VEGF, CD31, $\beta$-catenin and ALP, indicating that BSHX formula could restore the inhibited $\beta$-catenin and VEGF sinaling caused by OVX surgery.

\section{Discussion}

With the progress of the aging population in society, PMOP has became a frequent public health disease ${ }^{18}$. BSHX formula, severing as a complementary and alternative medicine, present an anti-PMOP effects in clinic and can prevent bone loss in OVX mice ${ }^{8}$, but its mechanism remains unclear. Here, we aimed to explore the underlying mechanism of BSHX formula acting on PMOP through network pharmacology and experiment approaches.

Based on multi-database mining and co-targets screening between BSHX formula and PMOP, a total of 64 overlapping genes were preliminarily obtained and regarded as the potential action mechanism of BSHX formula. PPI network analysis conformed 21 central gene targets, including VEGFA and CTNNB1 that is the key component of VEGF signaling and $\beta$-catenin signaling, respectively. Furthermore, the results of GO and KEGG enrichment analyses focused mainly on angiogenesis and cell proliferation, and these two biological processes were directly controlled by VEGF signaling and $\beta$-catenin signaling. Therefore, by utilizing network pharmacology, we speculated that BSHX formula exacts anti-PMOP effects mainly through promoting angiogenesis and cell proliferation in a VEGF- and $\beta$-catenin-mediated manner.

The dynamic balance between bone formation and resorption maintains adult skeletal health ${ }^{19}$. Bone formation tends to weak due to the deficiency of estrogen, and PMOP occurs when bone formation directed by osteoblasts is slower than osteoclast-mediated bone resorption ${ }^{20}$. Osteoblasts, bone forming cells, play an important role in osteogenesis through self-proliferation and producing extracellular matrix ${ }^{21}$. Specific proteins associated with osteoblast state, such as ALP, can be largely secreted during cell proliferation and matrix maturation, regarding as an early phenotypic marker for osteoblastogenesis $^{22}$. It is well known that bone is a highly vascularized tissue with a wide network of capillaries and blood vessels. Bone formation needs a spatial and temporal involvement of vascularization to provide essential nutrients, oxygen and growth factors, which is named osteogenesisangiogenesis coupling ${ }^{23,24}$. More remarkably, recent researches demonstrated that new blood vessels can directly regulate osteoblast proliferation for osteogenesis ${ }^{25,26}$. Therefore, activating osteogenesisangiogenesis coupling in bone might be an underlying strategy in the prevention of PMOP.

In the experimental validation part, we observed that OVX-induced bone loss were significantly rescued by BSHX formula, as shown by the increased BMD, BV/TV, Tb.Th and Tb.N and decreased Tb.Sp in BSHX formula treated mice. The results of $\mathrm{ABH}$ staining showed sparse trabeculae and blood vessels in the bone marrows of OVX mice, while both trabecular bone area and blood vessel number were largely improved after treatment with BSHX formula. Furthermore, we found higher levels of ALP and CD31 in the 
BSHX formula treated mice compared to the OVX ones. All these data indicated that BSHX formula preserves bone mass mainly through promoting osteogenesis-angiogenesis coupling.

Indeed, multiple anabolic signaling pathways positively control osteogenesis and angiogenesis, especially WNT and VEGF pathways. Canonical WNT/ $\beta$-catenin signaling has been reported to promote proliferation and differentiation of osteoblasts ${ }^{27,28}$. Its inhibition exerts critical effects in the development of $\mathrm{PMOP}^{29}$. VEGF, a major driver of angiogenesis, can activate a series of well-orchestral vascularization processes including endothelial cell proliferation, migration, sprouting vessel pruning and anastomosis, when it binds to the VEGF receptor on vascular endothelial cells ${ }^{30}$. Moreover, there is a close crosstalk between VEGF and $\beta$-catenin signaling in osteogenesis. The increased VEGF signaling can enhance $\beta$ catenin activity, resulting in excessively bone formation ${ }^{31}$. Thus, these two pathways are important molecular targets to prevent the progression of PMOP. Combined with KEGG enrichment analysis, we further determined the changes of $\beta$-catenin and VEGF signaling pathways in the OVX mice by $\mathrm{IHC}$, and the results showed that the deceased $\beta$-catenin and VEGF proteins in the OVX mice were significantly restored by BSHX formula. All these data indicated that BSHX formula exerted anti-PMOP effects primarily through $\beta$-catenin-mediated osteogenesis and VEGF-mediated angiogenesis.

\section{Conclusions}

In summary, through network pharmacology and an animal experimental validation, our present study revealed that BSHX formula attenuates PMOP by promoting osteogenesis and angiogenesis in a VEGFand $\beta$-catenin-mediated manner. We also provided a new alternative for the clinical treatment of PMOP.

\section{Abbreviations}

ABH, Alcian Blue Hematoxylin/Orange G; ALP, alkaline phosphatase; BSHX, Bushenhuoxue; BP, biological process; $\mathrm{BMD}$, bone mineral density; CC, cellular component; GO, gene ontology; KEGG, Kyoto Encyclopedia of Genes and Genomes; MF, molecular function; Micro-CT, micro-computed tomography; OVX, ovariectomized; PMOP, postmenopausal osteoporosis; PPI, protein-protein interaction; TCM, Traditional Chinese medicine; TCMSP, Traditional Chinese Medicine System Pharmacology Database; TCMID, Traditional Chinese Medicines Integrated Database, VEGF, vascular endothelial growth factor.

\section{Declarations}

\section{Conflicts of interest}

All authors state that they have no conflicts of interest.

\section{Funding statement}

This study was supported by Traditional Chinese Medical Administration of Zhejiang Province (grant nos. 2021ZQ082, 2021ZA131). 


\section{Author's contributions}

Study conception and design: Chenjie Xia, Liang Fang. Acquisition of data: Chunhai Ke, Yizhou Chen, Yi Wang, Dandan Mao, Guonong He. Analysis and interpretation of data: Chengliang Wu, Liang Fang, Yizhou Chen, Hongkai Lou, Hongting Jin. Drafting the article or revising it critically for important intellectual content: Chenjie Xia, Chengliang Wu. Final approval of the version of the article to be published: Chenjie Xia, Chunhai Ke, Chengliang Wu.

\section{References}

1. Compston, J. E., McClung, M. R. \& Leslie, W. D. Osteoporosis. Lancet 393, 364-376 (2019).

2. Wright, N. C., Looker, A. C., Saag, K. G., Curtis, J. R., Delzell, E. S., Randall, S. \& Dawson-Hughes, B. The recent prevalence of osteoporosis and low bone mass in the United States based on bone mineral density at the femoral neck or lumbar spine. J Bone Miner Res 29, 2520-2526 (2014).

3. Yuan, Y., Yang, L., Liu, T., Zhang, H. \& Lu, Q. Osteoclastogenesis inhibition by mutated IGSF23 results in human osteopetrosis. Cell Prolif 52, e12693 (2019).

4. Langdahl, B. L. Overview of treatment approaches to osteoporosis. Br J Pharmacol (2020).

5. Khosla, S. \& Hofbauer, L. C. Osteoporosis treatment: recent developments and ongoing challenges. Lancet Diabetes Endocrinol 5, 898-907 (2017).

6. Liu, Y., Liu, J. P. \& Xia, Y. Chinese herbal medicines for treating osteoporosis. Cochrane Database Syst Rev CD005467 (2014).

7. Hu, S. et al. Bushenhuoxue formula accelerates fracture healing via upregulation of TGF- $\beta /$ Smad2 signaling in mesenchymal progenitor cells. Phytomedicine 76, 153256 (2020).

8. Xia, C. et al. Bushenhuoxue formula promotes osteogenic differentiation of growth plate chondrocytes through $\beta$-catenin-dependent manner during osteoporosis. Biomed Pharmacother 127, 110170 (2020).

9. Wang, S. J., Yue, W., Rahman, K., Xin, H. L., Zhang, Q. Y., Qin, L. P. \& Zhang, H. Mechanism of Treatment of Kidney Deficiency and Osteoporosis is Similar by Traditional Chinese Medicine. Curr Pharm Des 22, 312-320 (2016).

10. Hopkins, A. L. Network pharmacology. Nat Biotechnol 25, 1110-1111 (2007).

11. Hopkins, A. L. Network pharmacology: the next paradigm in drug discovery. Nat Chem Bio/ 4, 682-690 (2008).

12. Li, S. \& Zhang, B. Traditional Chinese medicine network pharmacology: theory, methodology and application. Chin J Nat Med 11, 110-120 (2013).

13. Zhang, R., Zhu, X., Bai, H. \& Ning, K. Network Pharmacology Databases for Traditional Chinese Medicine: Review and Assessment. Front Pharmacol 10, 123 (2019).

14. Wang, P. E. et al. Bushenhuoxue formula attenuates cartilage degeneration in an osteoarthritic mouse model through TGF- $\beta /$ MMP13 signaling. J Trans/ Med 16, 72 (2018). 
15. Zhu, N. \& Hou, J. Exploring the mechanism of action Xianlingubao Prescription in the treatment of osteoporosis by network pharmacology. Comput Biol Chem 85, 107240 (2020).

16. Xia, C. et al. Activation of $\beta$-catenin in Col2-expressing chondrocytes leads to osteoarthritis-like defects in hip joint. J Cell Physiol 234, 18535-18543 (2019).

17. Xia, C. et al. TGF- $\beta /$ Smad2 signalling regulates enchondral bone formation of Gli1+ periosteal cells during fracture healing. Cell Prolif e12904 (2020).

18. Liu, X., Liu, H., Xiong, Y., Yang, L., Wang, C., Zhang, R. \& Zhu, X. Postmenopausal osteoporosis is associated with the regulation of SP, CGRP, VIP, and NPY. Biomed Pharmacother 104, 742-750 (2018).

19. Guo, L. et al. Estrogen inhibits osteoclasts formation and bone resorption via microRNA-27a targeting PPARy and APC. J Cell Physio/ 234, 581-594 (2018).

20. Kiernan, J., Davies, J. E. \& Stanford, W. L. Concise Review: Musculoskeletal Stem Cells to Treat AgeRelated Osteoporosis. Stem Cells Trans/ Med 6, 1930-1939 (2017).

21. Komori, T. Regulation of Proliferation, Differentiation and Functions of Osteoblasts by Runx2. Int $J$ Mol Sci 20, (2019).

22. Taufik, N. H., Tulaar, A., Moesbar, N. \& Ganie, R. A. The Effect of Isometric Exercise Plantar Flexor on Osteoblast Activity Measured by Bone Specific Alkaline Phosphatase and Callus Formation in a Patient Post Open Reduction Internal Fixation with Non-articular Tibia Fracture. Open Access Maced J Med Sci 7, 3409-3415 (2019).

23. Kusumbe, A. P., Ramasamy, S. K. \& Adams, R. H. Coupling of angiogenesis and osteogenesis by a specific vessel subtype in bone. Nature 507, 323-328 (2014).

24. Schipani, E., Maes, C., Carmeliet, G. \& Semenza, G. L. Regulation of osteogenesis-angiogenesis coupling by HIFs and VEGF. J Bone Miner Res 24, 1347-1353 (2009).

25. Peng, Y., Wu, S., Li, Y. \& Crane, J. L. Type H blood vessels in bone modeling and remodeling. Theranostics 10, 426-436 (2020).

26. Xie, H. et al. PDGF-BB secreted by preosteoclasts induces angiogenesis during coupling with osteogenesis. Nat Med 20, 1270-1278 (2014).

27. Choi, J. Y., Lai, J. K., Xiong, Z. M., Ren, M., Moorer, M. C., Stains, J. P. \& Cao, K. Diminished Canonical $\beta$-Catenin Signaling During Osteoblast Differentiation Contributes to Osteopenia in Progeria. $J$ Bone Miner Res 33, 2059-2070 (2018).

28. Mulati, M. et al. The long noncoding RNA Crnde regulates osteoblast proliferation through the Wnt/ $\beta$ catenin signaling pathway in mice. Bone 130, 115076 (2020).

29. MacDonald, B. T., Tamai, K. \& He, X. Wnt/beta-catenin signaling: components, mechanisms, and diseases. Dev Cell 17, 9-26 (2009).

30. Herbert, S. P. \& Stainier, D. Y. Molecular control of endothelial cell behaviour during blood vessel morphogenesis. Nat Rev Mol Cell Biol 12, 551-564 (2011).

31. Maes, C. et al. Increased skeletal VEGF enhances beta-catenin activity and results in excessively ossified bones. EMBO J 29, 424-441 (2010). 


\section{Figures}

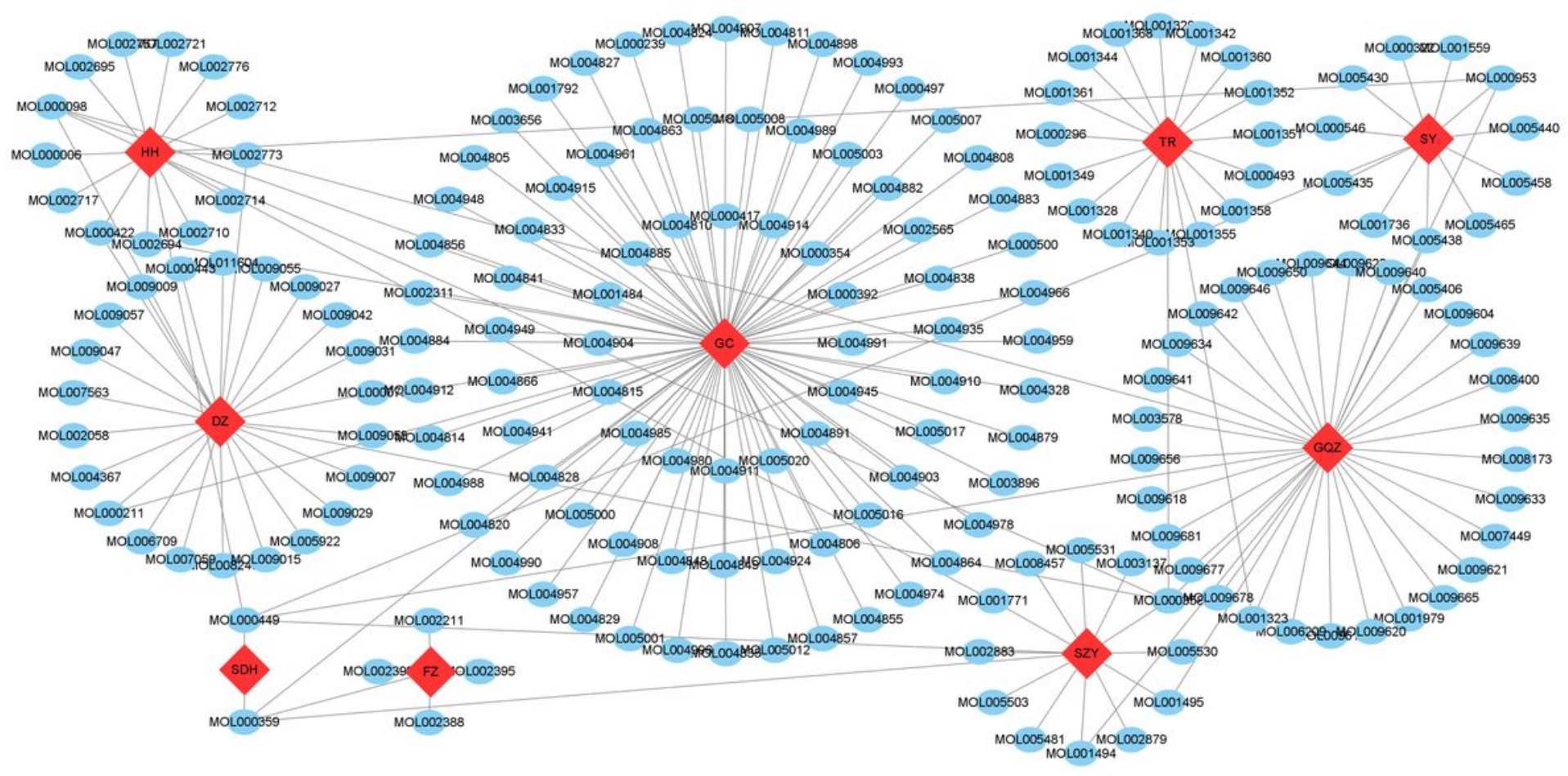

\section{Figure 1}

The network of Herb-Ingredient connection. The red square nodes represent herbs in BSHX formula, and the blue circles represent active ingredients. The edges represent the direct relationship between them. 


\section{BSHX formula}

\section{PMOP}

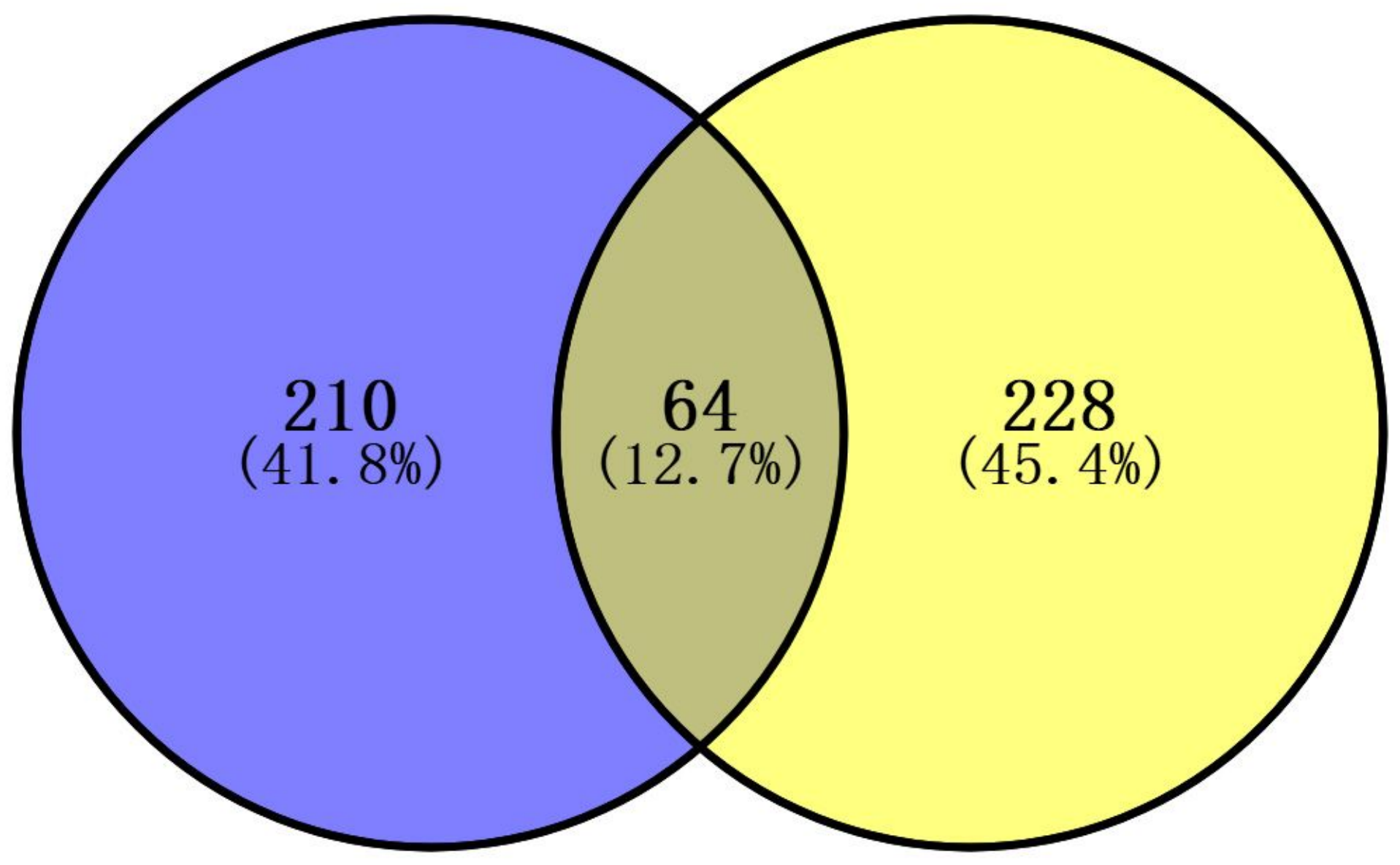

Figure 2

The Venn diagram of the gene targets both in BSHX formula and PMOP. The overlap part means the underlying therapeutic targets through which BSHX formula treats PMOP. 


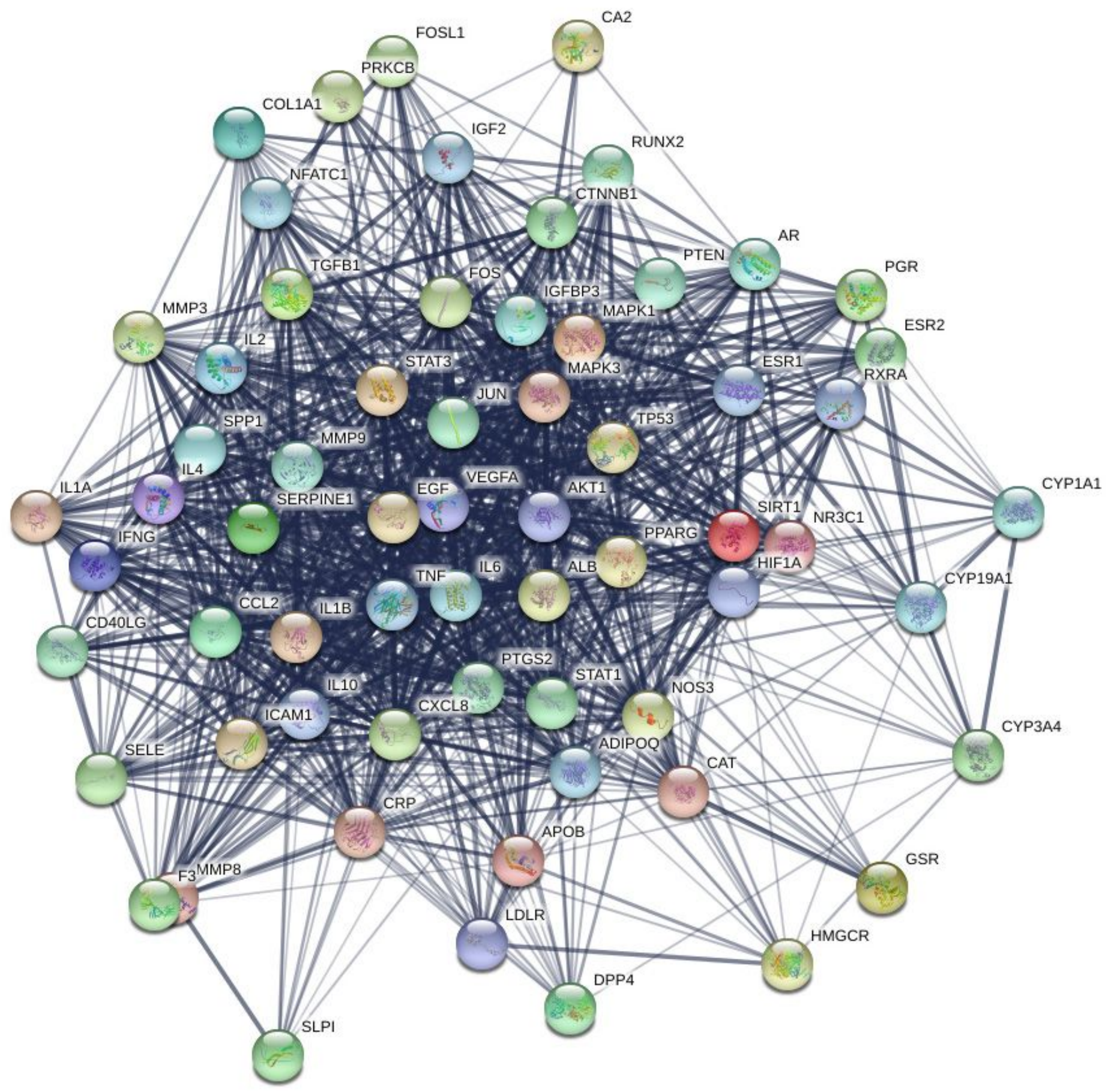

Figure 3

PPI network construction based on String database. Line thickness indicates the confidence level of the supporting data. 


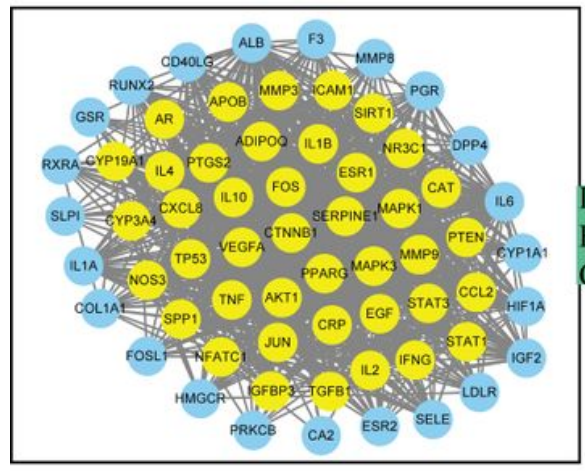

64 nodes and 1110 edges

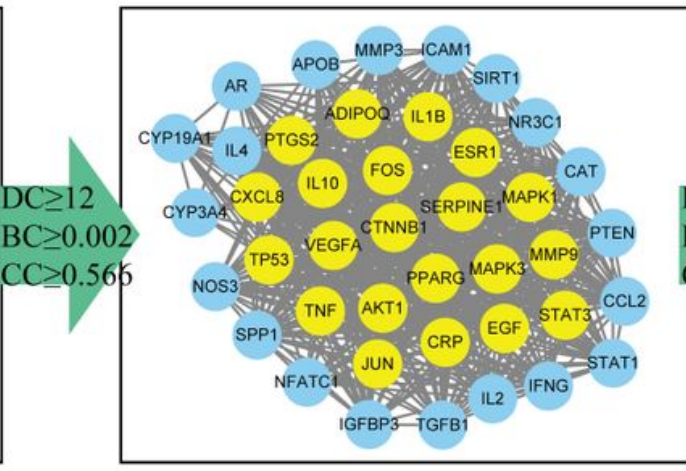

41 nodes and 634 edges

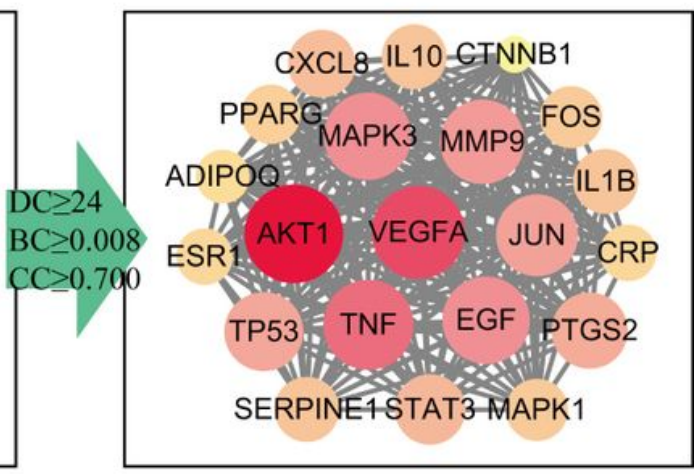

21 nodes and 202 edges

\section{Figure 4}

The topological screening process of the PPI network. In the third image, the bigger size and more brilliant color represent higher DC value.

A

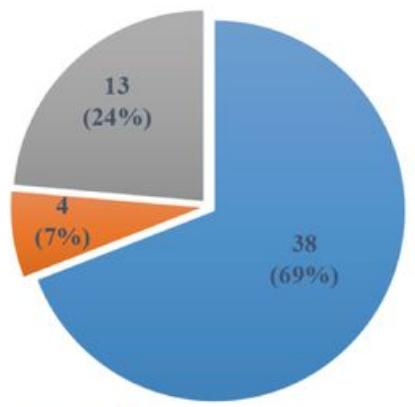

- GOTERM_BP $\square$ GOTERM_CC 1 GOTERM_MF
B

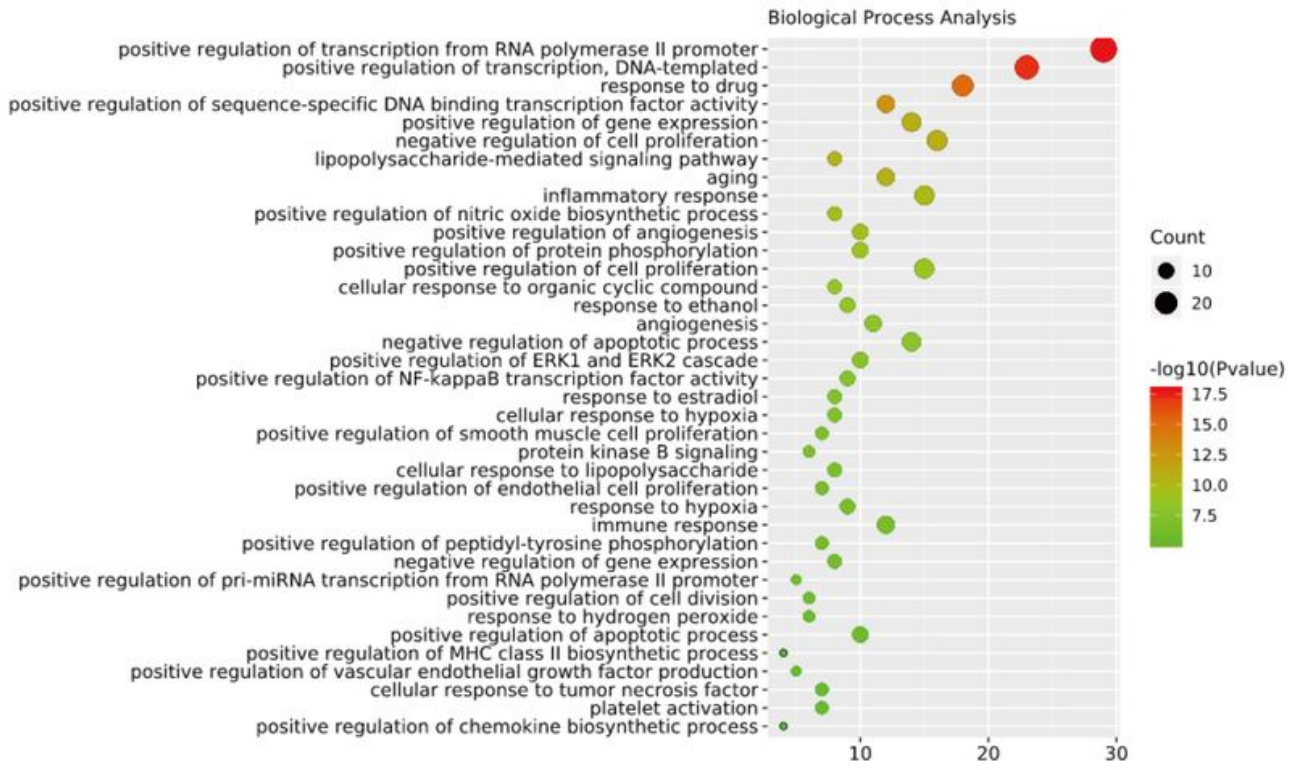

\section{Figure 5}

GO enrichment analysis of 64 overlapping targets. (A) Three types of GO items in DAVID database (Blue, GOTRIM_BP: biological process. Yellow, GOTRIM_CC: cellular component. Gray, GOTRIM_MF: molecular function). (B) The bubble diagram of BP items. 38 BP items were arranged in the descending order of Pvalue. 


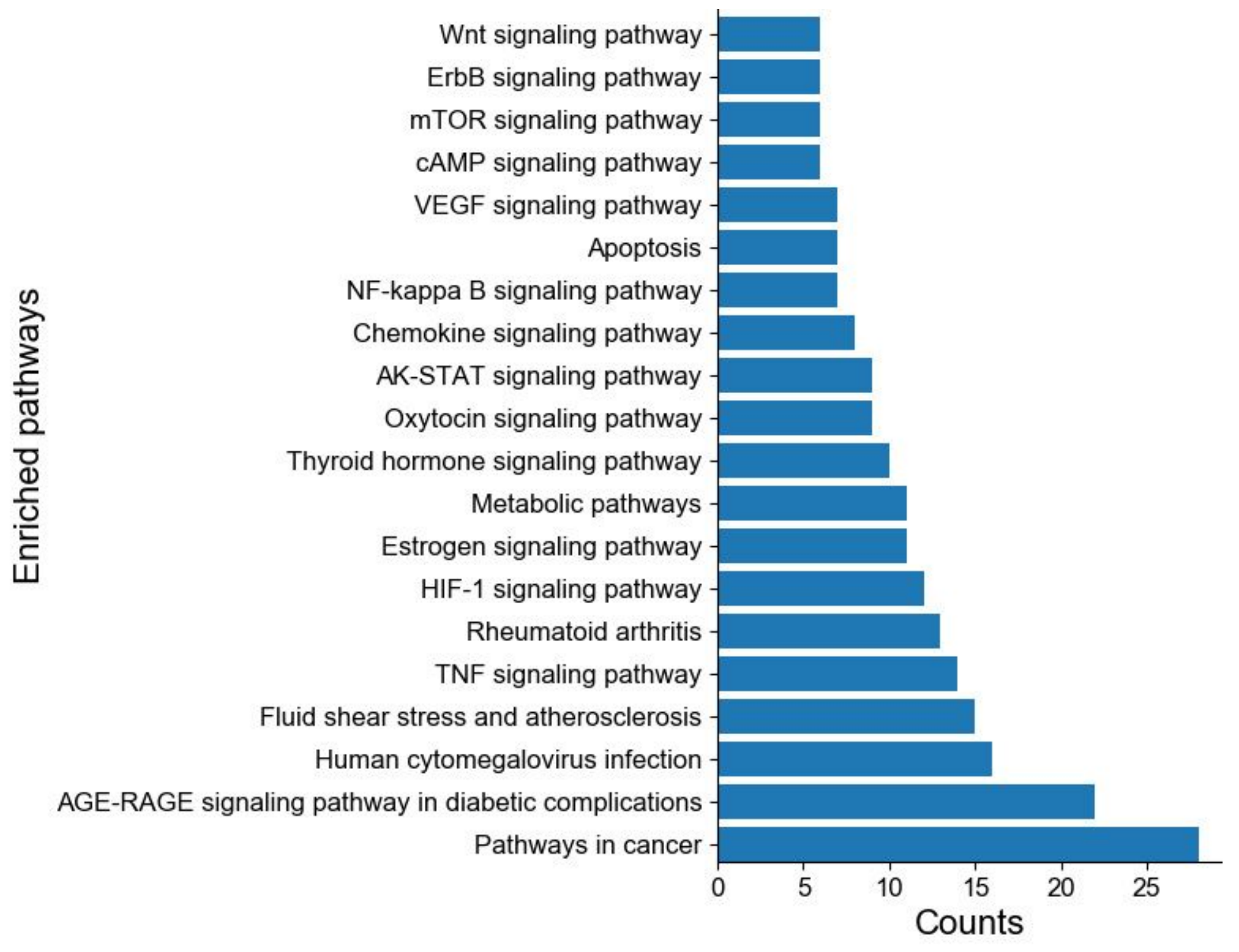

Figure 6

KEGG enrichment analysis of 64 overlapping genes. The image showed the details of 20 pathways. 
A

Sham
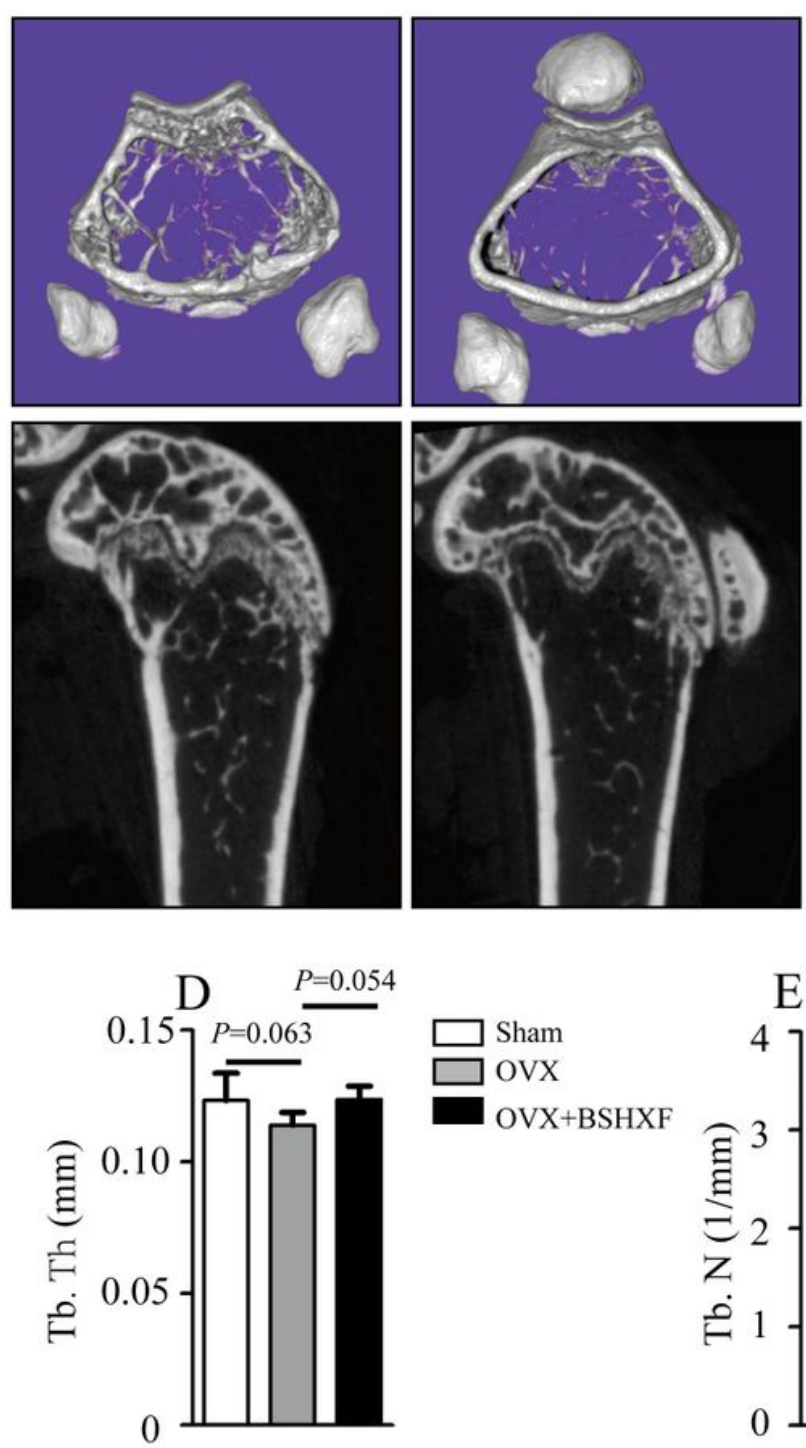

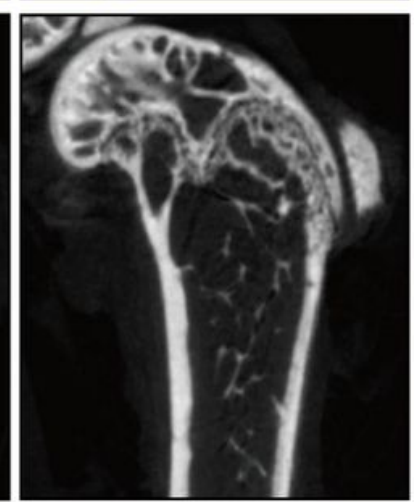

BSHX formula
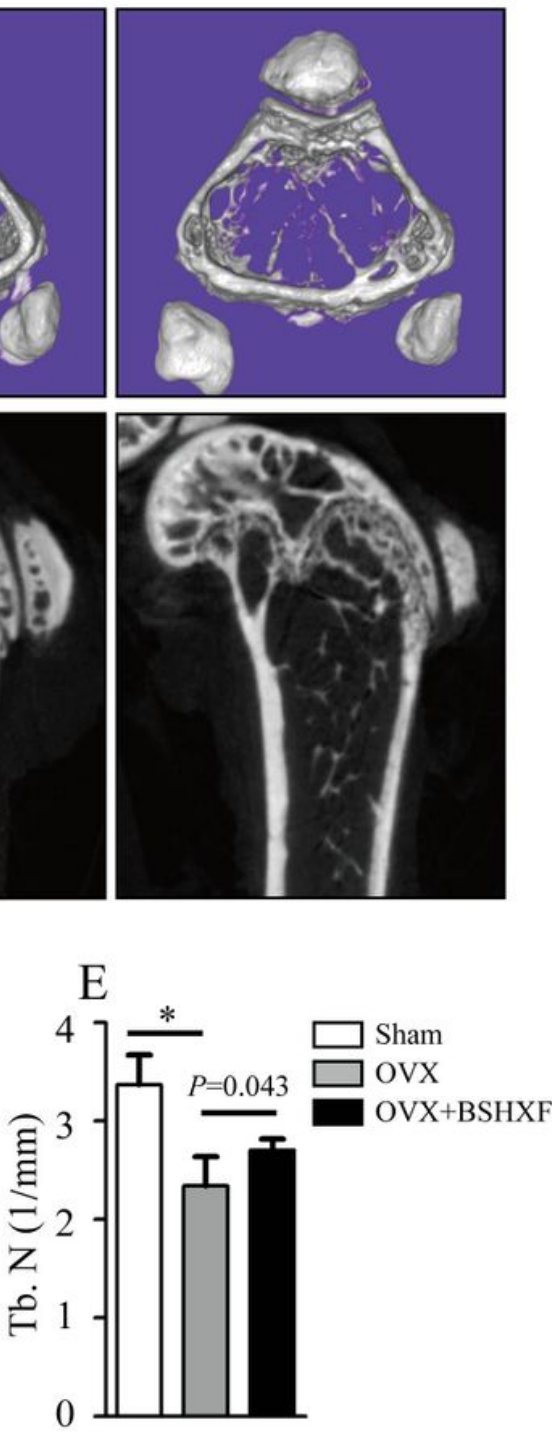

B
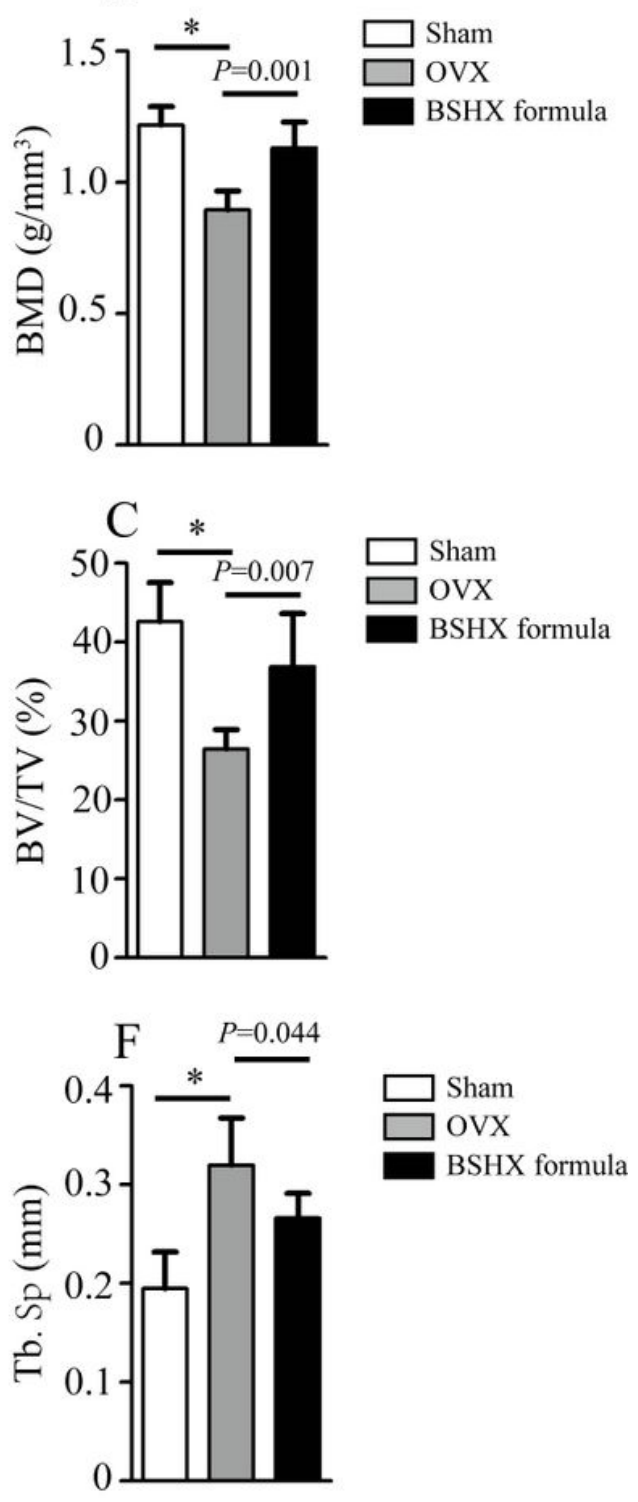

Figure 7

BSHXF protects against bone loss in OVX mice. (A) Representative $\mu C T$ images. Quantification of microstructural parameters including BMD (B), BV/TV (C), Tb. Th (D), Tb. N (E) and Tb. Sp (F). *P $<0.001$. 
A
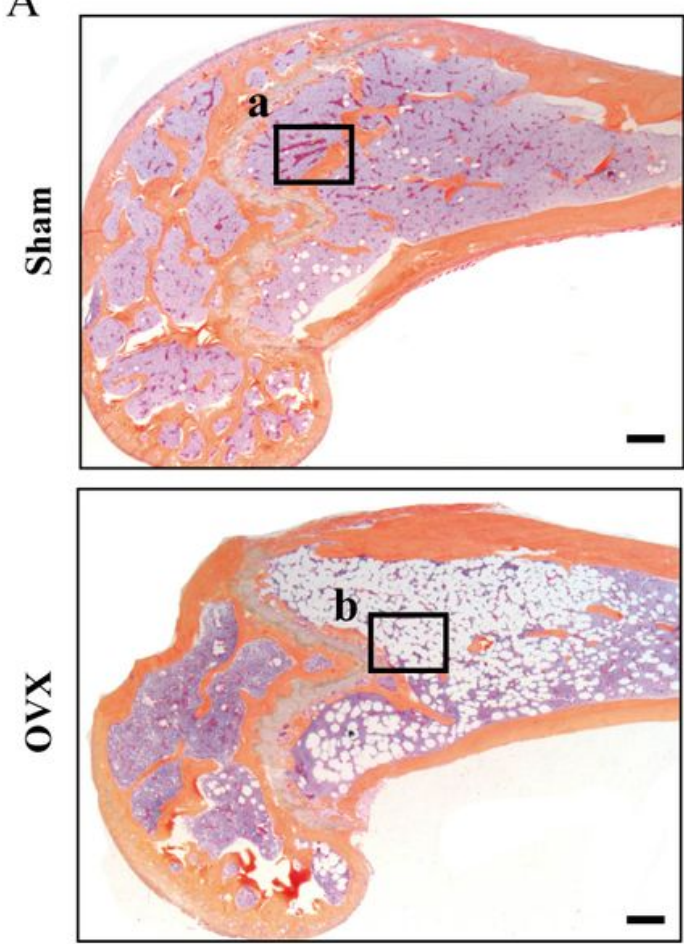

ํㅜㄹ

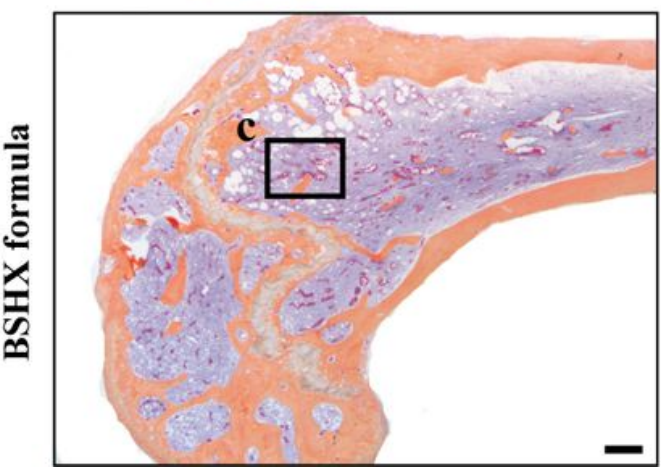

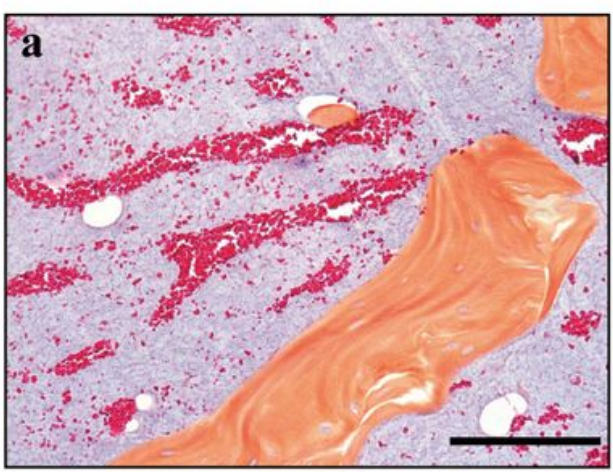
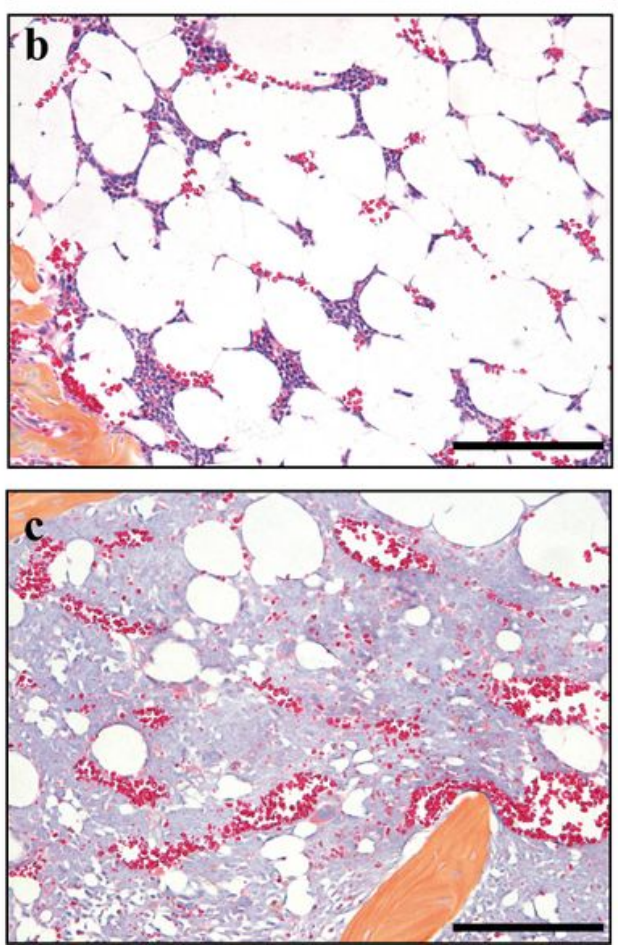
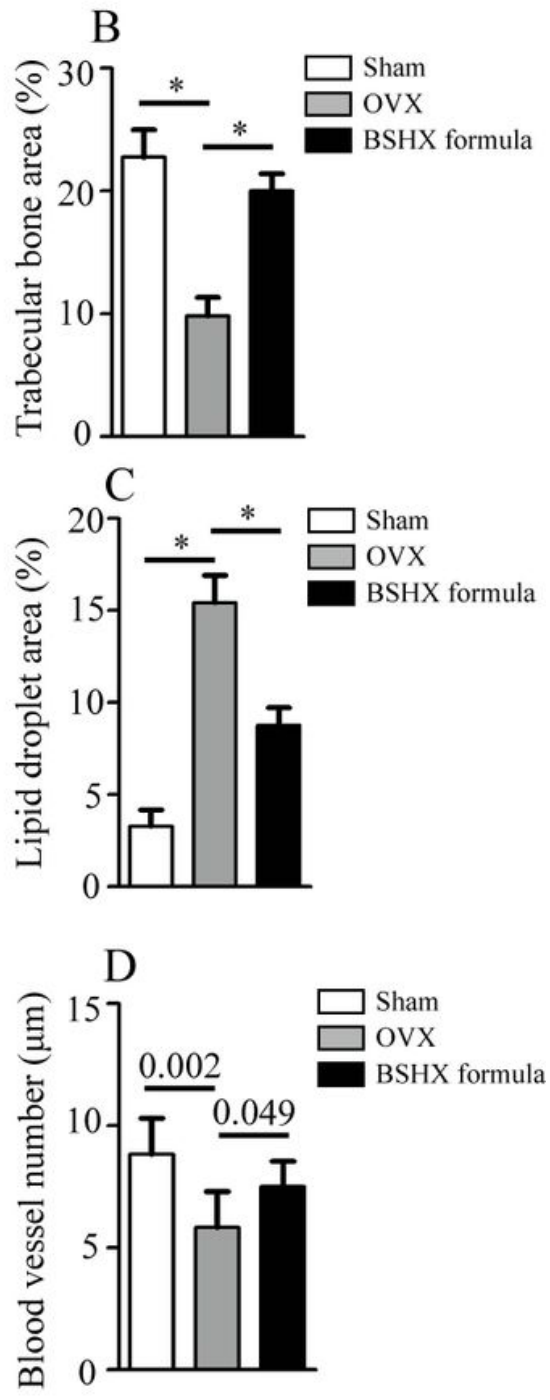

Figure 8

Changes of trabecular bone, lipid droplet and blood vessel in OVX mice. (A) Alcian Blue Hematoxylin/Orange $\mathrm{G}$ staining of distal femur. a-c: boxed areas at a high magnification. Orange: trabecular bone; White: lipid droplet; Red: blood vessel. (B) The area of trabecular bone (\%). (C) The area of lipid droplet (\%). (D) The number of blood vessel. Scale bars: $1000 \mu \mathrm{m}$. ${ }^{*}<0.001$. 
A

Sham
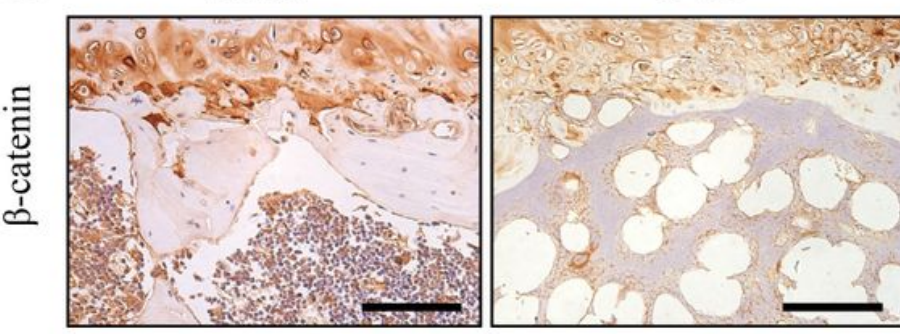

B
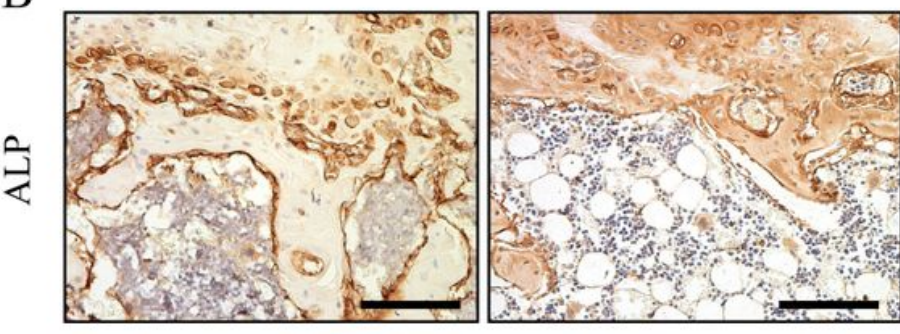

$\mathrm{C}$
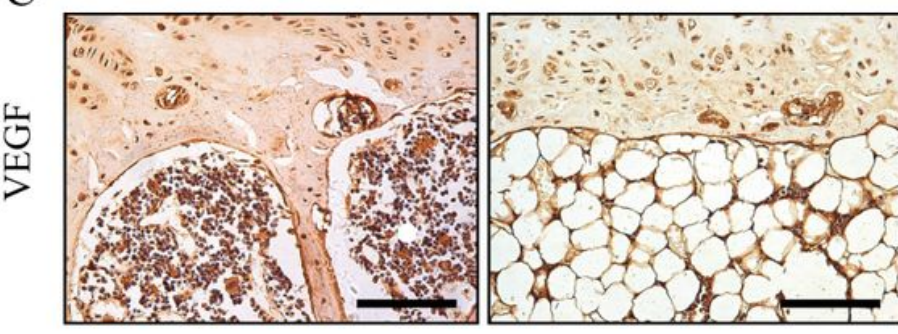

$\mathrm{D}$

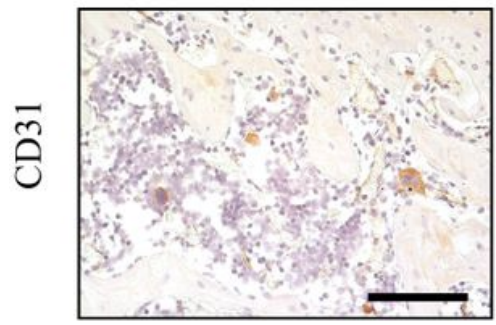

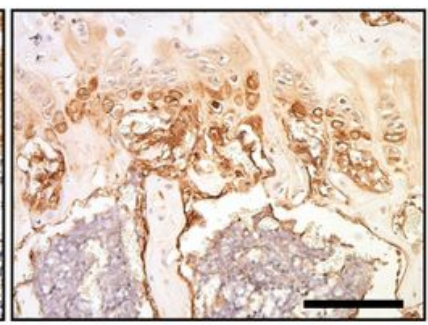

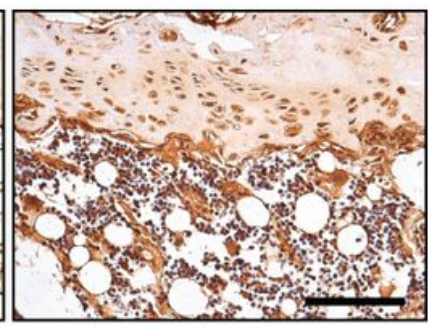

BSHX formula
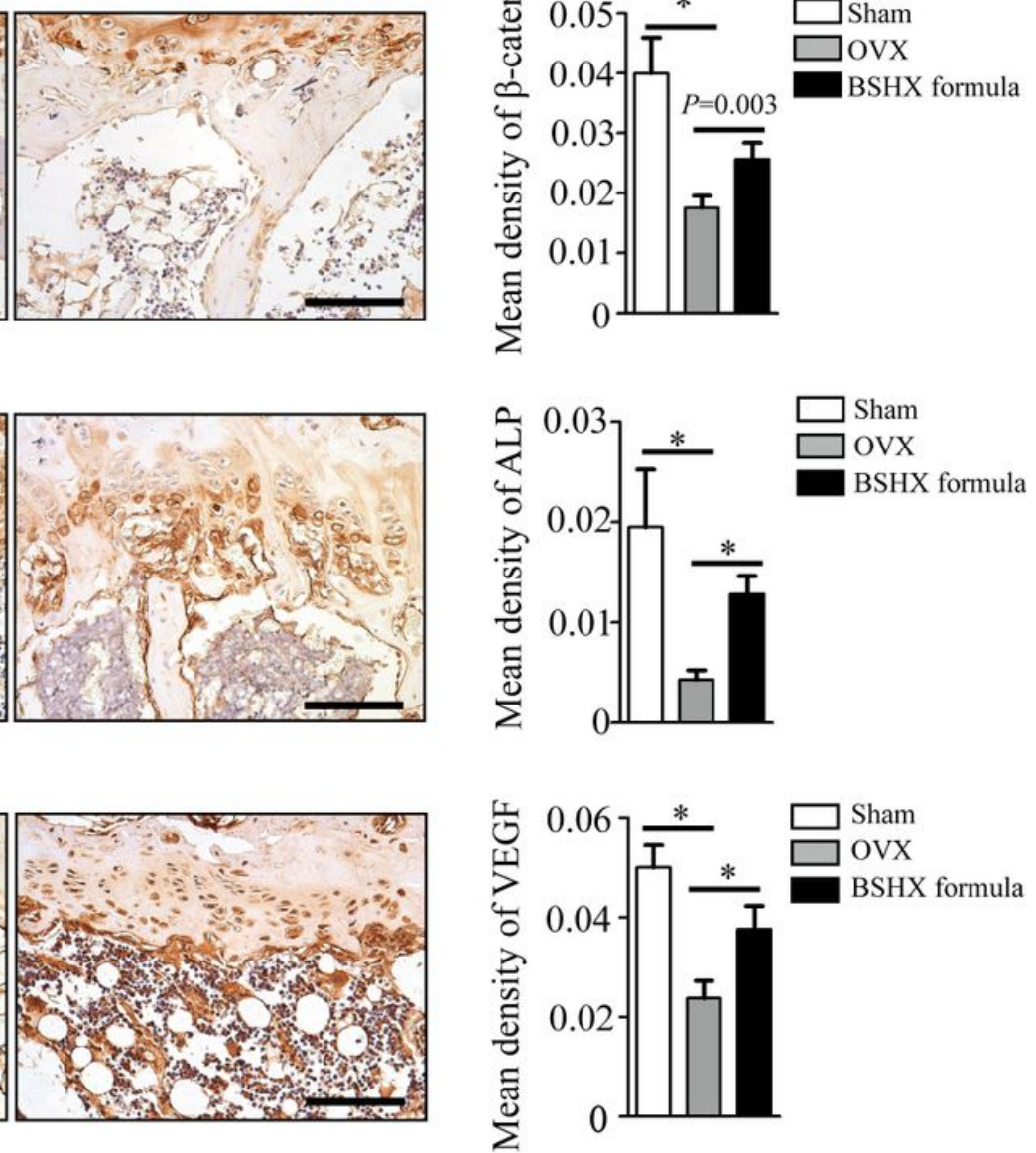

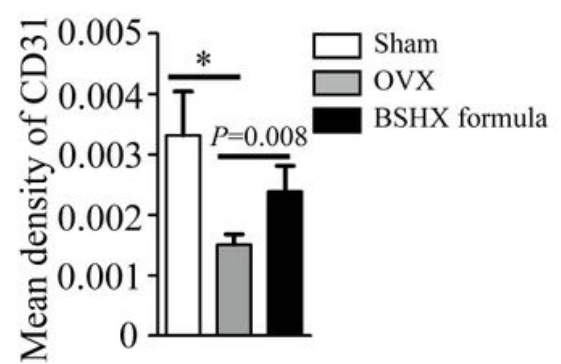

Figure 9

Down-regulation of $\beta$-catenin and VEGF signaling were restored by BSHX formula. Representative IHC images and quantifications of $\beta$-catenin (A), ALP (B), VEGF (C) and CD31 (D). Scale bars: $1000 \mu \mathrm{m}$. *P< 0.001 . 